\title{
The frequency of osteolytic bone metastasis is determined by conditions of the soil, not the number of seeds; evidence from in vivo models of breast and prostate cancer
}

\author{
Ning Wang ${ }^{1 *}$, Kimberley J. Reeves ${ }^{3}$, Hannah K. Brown ${ }^{1}$, Anne C M Fowles ${ }^{1}$, Freyja E. Docherty ${ }^{1}$,
}

Penelope D. Ottewell ${ }^{2}$, Peter I. Croucher ${ }^{4}$, Ingunn Holen ${ }^{2}$ and Colby L. Eaton ${ }^{1}$

\begin{abstract}
Background: While both preclinical and clinical studies suggest that the frequency of growing skeletal metastases is elevated in individuals with higher bone turnover, it is unclear whether this is a result of increased numbers of tumour cells arriving in active sites or of higher numbers of tumour cells being induced to divide by the bone micro-environment. Here we have investigated how the differences in bone turnover affect seeding of tumour cells and/or development of overt osteolytic bone metastasis using in vivo models of hormone-independent breast and prostate cancer.

Methods: Cohorts of 6 (young) and 16 (mature)-week old BALB/C nude mice were culled 1, 7 and 21 days after received intracardiac injection of luciferase expressing human prostate (PC3) or breast cancer (MDA-MB-231) cell lines labelled with a fluorescent cell membrane dye (Vybrant DiD). The presence of growing bone metastases was determined by bioluminescence using an in vivo imaging system (IVIS) and followed by anatomical confirmation of tumour metastatic sites post mortem, while the presence of individual fluorescently labelled tumour cells was evaluated using two-photon microscopy ex vivo. The bone remodelling activities were compared between young and mature naïve mice (both male and female) using micro-CT analysis, ELISA and bone histomorphometry.

Results: Both prostate and breast cancer cells generated higher numbers of overt skeletal lesions in young mice $(\sim 80 \%)$ than in mature mice ( $20 \%)$. Although mature mice presented with fewer overt bone metastases, the number of tumour cells arriving/colonizing in the tibias was comparable between young and mature animals. Young naïve mice had lower bone volume but higher bone formation and resorption activities compared to mature animals.

Conclusions: Our studies suggest that higher frequencies of growing osteolytic skeletal metastases in these models are linked to increased bone turnover and not to the initial number of tumour cells entering the bone microenvironment.
\end{abstract}

Keywords: Tumor-induced bone disease, Bone remodelling, Two-photon microscopy, Animal models

\footnotetext{
* Correspondence: n.wang@sheffield.ac.uk

${ }^{1}$ The Mellanby Centre for Bone Research, Department of Human Metabolism, Medical School, University of Sheffield, Beech Hill Road, Sheffield S10 2RX, UK Full list of author information is available at the end of the article
} 


\section{Background}

About $90 \%$ patients with advanced breast and prostate cancer have incurable bone metastases [1, 2], with a mean survival of one year $[3,4]$. Treatments that aim to either prevent or suppress the growth of bone metastasis are limited. Anti-bone resorptive agents such as zoledronic acid, prevent bone loss and skeletal-related events (SREs), but do not increase survival in unselected patients with advanced disease [4-7]. However, a better understanding of the early mechanisms leading to the development of bone metastasis would allow such therapies to be specifically targeted to early lesions as they arise.

The 'seed and soil hypothesis' for metastasis proposes that only the right cells disseminated from primary tumour (the 'seeds') landing in the right microenvironment (the 'soil') can form secondary (metastatic) tumours successfully [8]. It has been suggested that prostate and breast cancers contain a small percentage $(<1 \%)$ of stem cell-like populations and that it is these cells that have the potential to act as the 'seeds', leaving the primary site, surviving in the circulation, homing into the bone, where they eventually form bone metastases [9-14]. In this context, bone is the fertile 'soil', providing a supportive microenvironment ('bone metastasis niche') for tumour cell survival and secondly, under the right conditions, for the proliferation of clinically relevant metastases. New evidence has shown that disseminated prostate cancer cells target the haemopoietic stem cell niche to establish footholds in bone, with the osteoblastic lineages as the key components of the niche [15, 16]. In addition, bone continuously renews itself with precisely balanced osteoblastic bone formation and osteoclastic bone resorption. Elevated levels of bone turnover have been shown to be correlate with increased numbers of metastases, in various xenograft models and clinical studies [17-22]. Whether this effect is a result of different bone environments increasing tumour cell seeding, or of environments inducing proliferation of resident tumour cells in bone to form overt metastases, remains to be determined.

In this study, we investigated the initiation of prostate and breast cancer bone metastases in young (6-week old) and in mature (16-week old) athymic mice. Both bone turnover rates and the frequency of metastases were lower in the mature compared to the younger animals allowing us to study the initiation of metastases in different bone microenvironments. We used two-photon microscopy to identify initial seeding of tumour cells into bone and compared this with the frequency of growing metastases. This is the first study to directly test the hypothesis that the frequency of bone metastasis is equally dependent on numbers of seeded tumour cells and growth induction by the bone microenvironment.

\section{Methods}

Mice

All studies were performed using 6-week or 16-week old BALB/cAnNCrl immunocompromised (athymic nude) mice (Charles River, Kent, UK) as xenograft models of bone metastasis. All procedures complied with the UK Animals (Scientific Procedures) Act 1986 and were reviewed and approved by the local Research Ethics Committees of the University of Sheffield under Home Office project licence 40/3462 (Sheffield, UK).

\section{Cell lines}

The human prostate cancer cell line PC3 (ATCC, Middlesex, UK) and human breast cancer cell line MDA-MB-231 (ATCC, Middlesex, UK) were stably transfected with a firefly luciferase gene luc2 (pGL4.51 [luc2/CMV/Neo] vector, Promega, Southampton, UK) and denoted as PC3-NW1 and MDA-MB-231 luc2. Both cell lines were maintained in Dulbecco's Modified Eagle Medium (DMEM) (Life Technologies, Paisley, UK), supplemented with 100 Units/mL Penicillin, $100 \mu \mathrm{g} / \mathrm{mL}$ Streptomycin and $10 \%$ foetal calf serum (FCS) (Sigma Aldrich Co Ltd, Poole, UK).

\section{Xenograft models}

Human cancer cells were initially stained with $5 \mu \mathrm{M}$ lipophilic carbocyanine dye Vybrant DiD (Life Technologies, Paisley, UK) according to the manufacture's protocol. Using the Vybrant DiD dye, which is lost as cells divide, allows non-dividing/slowly dividing cells to be identified and distinguished from proliferating cells [15, 23 , 24]. A single-cell suspension of $1 \times 10^{5} \mathrm{DiD}$ labelled PC3-NW1 cells $/ 100 \mu \mathrm{L}$ PBS and $0.75 \times 10^{5}$ DiD labelled MDA-MB-231 luc2 cells/100 $\mu \mathrm{L}$ PBS were injected into the left cardiac ventricle (intracardiac (i.c.) injection) of 6- or 16-week old male and female BALB/c nude mice, respectively. Overt tumours were monitored up to 8 weeks post injection using an in vivo imaging system (IVIS, PerkinElmer, Cambridge, UK) and the tumour burden was measured based on radiance of luminescence using the Living Image software (PerkinElmer), followed by anatomical confirmation of tumour metastatic sites post mortem. Cohorts of animals (minimum $n=6$ /group) were euthanized on day 1,7 and 21 post injection and individual non-dividing DiD labelled tumour cells were quantified in the tibiae ex vivo by two-photon microscopy, to understand the quantity of tumour cells arriving and colonizing within the bone marrow.

\section{Two-photon microscopy}

Dissected right tibiae were prepared and sectioned as described previously [15, 24]. An area of $2104 \mu \mathrm{m} \times$ $2525 \mu \mathrm{m}$ below the growth plate with $100 \mu \mathrm{m}$ in depth 
was imaged using a Zeiss LSM510 NLO upright multiphoton/confocal microscope (Carl Zeiss Inl, Cambridge, UK). A $633 \mathrm{~nm} \mathrm{HeNe}$ laser was used to detect DiD labelled cells while the bone was detected using the $900 \mathrm{~nm}$ Chameleon multiphoton laser. The number of tumour cells (DiD positive events) and their distance to the nearest bone surface (a parameter indicating the location of tumour cells towards osteoblastic niche) was analysed using the Volocity 3D Image Analysis software 6.01 (PerkinElmer, Cambridge, UK).

\section{Micro-CT analysis}

Right femurs were dissected and scanned by SkyScan 1172 desktop micro-CT (SkyScan) at the resolution of 6 $\mu \mathrm{m}$. Trabecular morphometry, characterized as trabecular bone content (BV/TV) was measured from a $1.0 \mathrm{~mm}$ thick region $0.2 \mathrm{~mm}$ above the growth plate where metastatic tumour cells are generally situated. Nomenclature and symbols were used to describe the micro-CT derived bone morphometries according to the published guidelines [25].

\section{Serum bone remodelling markers}

Tartrate-resistant acid phosphatase 5b (TRAP) activity in mouse serum was determined as a measure of bone resorption activity using an IDS MouseTRAP ${ }^{\mathrm{m}}$ Assay (Immunodiagnostic Systems, Tyne \& Wear, UK). Type 1 procollagen amino-terminal-propeptide (P1NP) and osteocalcin were determined for bone formation activities, using a Rat/Mouse P1NP competitive immunoassay kit (Immunodiagnostic Systems, Tyne \& Wear, UK) and a Mouse Osteocalcin ELISA Kits (Takara Bio Europe, Saint Germain, France), for male and female mice samples, respectively.

\section{Bone histomorphometry}

Dissected left tibiae were prepared and TRAP stained as described previously [26]. The number of osteoblasts (N.Ob/B.Pm), the bone surface covered by osteoblasts (Ob.Pm/B.Pm), the number of osteoclasts (N.Oc/B.Pm), and the bone surface covered by osteoclasts (Oc.Pm/ B.Pm) were determined on a $1.5 \mathrm{~mm}$ length of endocortical surfaces, using a DMRB microscope (Leica Microsystems, Wetzlar, Germany) with the Osteomeasure bone histomorphometry software (OsteoMetrics, Inc. Decatur, GA, USA).

\section{Statistic analysis}

All data are expressed as mean \pm SEM. Statistical significance was tested for using an unpaired Student's t-test using the Prism 6 software (GraphPad, La Jolla, USA). $P<0.05$ was considered to be significant.

\section{Results}

The frequency of overt, skeletal prostate cancer metastases was elevated in young compare to mature male mice

We first established how prostate cancer cells formed tumours in bone following intra-cardiac injection, comparing young and mature male mice. Three weeks after tumour cell injection, young animals developed tumours, observed by bioluminescence, with an overall skeletal tumour frequency of greater than $80 \%$ in multiple experiments $(n=12$ in total). In contrast, only $20 \%$ of mature animals developed skeletal tumours $(n=12)$ three weeks post injection and $40 \%$ of mature animals developed skeletal tumours by eight weeks (Fig. 1a). The young mice had significantly higher number of skeletal tumours (3.4 \pm 0.4 tumours per mice vs $0.4 \pm 0.2, p<0.0001)$, but not non-skeletal tumours in soft tissue and organs, such as in skeletal muscle, liver, adrenal glands, except peri- or intra-cardial tumours caused by incorrect injections, compared to mature mice $(0.4 \pm 0.2$ vs $0.3 \pm 0.1$, $p=0.7314$ )(Fig. $1 \mathrm{~b}$ and $\mathrm{c}$ ). This was reflected by the total tumour burden determined by the radiance of tumour luminescence [37556 \pm 10797 vs $2754 \pm 1689$ photons/ $\mathrm{sec} / \mathrm{cm}^{2} /$ steradian $\left(\mathrm{p} / \mathrm{sec} / \mathrm{cm}^{2} / \mathrm{sr}\right.$ ), $p=0.0043$ ] (Fig. $1 \mathrm{~d}$ ). The skeletal tumours clearly caused osteolytic bone lesions in both young and old mice, as shown in the $3 \mathrm{D}$ micro-CT scanning image of a tumour bearing femur of young mice associated with bone destruction (Fig. 1e). This was confirmed by significant decrease in trabecular bone content (BV/TV) compared to non-tumour bearing femurs $(13.6 \pm 0.8$ vs $3.0 \pm 0.9$, $p<0.0001$ )(Fig. 1f).

\section{Seeding of prostate cancer cells to bone was not increased in young mice}

The higher frequency of tumours in bone could be due to increased numbers of tumour cells seeding in the niche in young animals. We therefore quantified the number of DiD-labelled prostate cancer cells in the tibia of male mice using two-photon microscopy ex vivo and found that increased tumour cell seeding was not responsible for this higher frequency of bone metastases observed in younger animals. DiD labelled prostate cancer cells were identified in tibial bone marrow of both younger and older mice at different time points ( 1 day, 7 days, and 3 weeks) following intra cardiac injection (Fig. 2a). Quantified using Volocity 3D image analysis software, the time course of tumour cells homing to the bone marrow showed a very similar pattern in younger and older mice, with the number of resident tumour cells reaching its peak 7 days post-injection. Significantly fewer tumour cells were present in younger mice on day 1 and at 3 weeks post-injection (Fig. 2b). Fewer cells were also observed in younger mice on day 7 but this failed to reach significance. Tumour cells were found 
a

a

Young
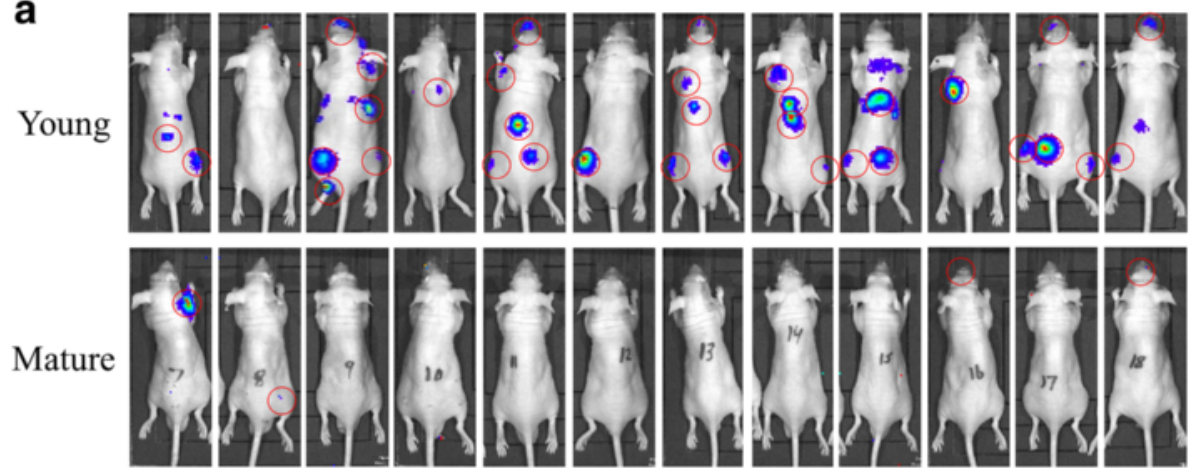

b

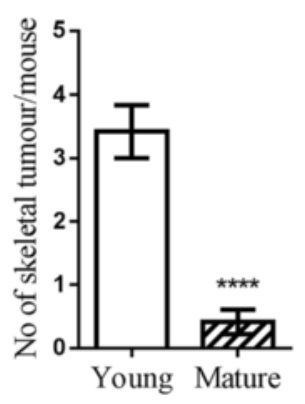

C

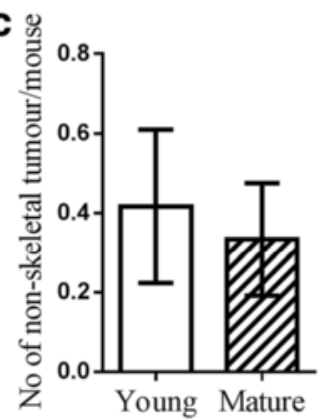

d

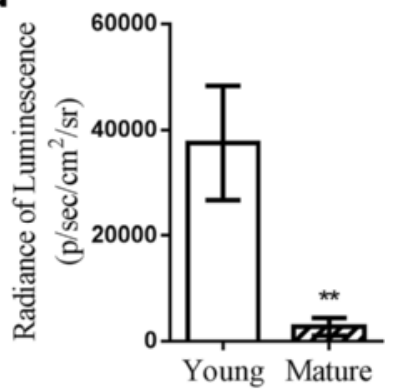

e

With tumour
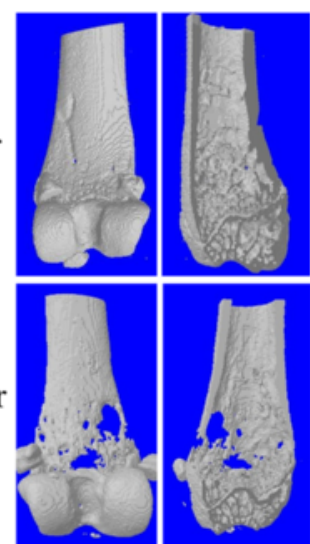

f

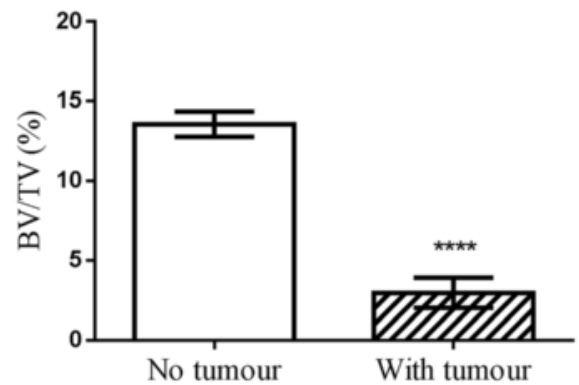

Fig. 1 Comparison of skeletal metastases by human prostate cancer cells in young and mature male mice. A single-cell suspension of $1 \times 10^{5} \mathrm{DiD}$ labelled PC3-NW1 cells/100 $\mu \mathrm{L}$ PBS was injected into the left cardiac ventricle of 6-week old (Young) or 16-week old (Mature) male BALB/c nude mice. a Tumour growth was monitored by in vivo imaging. Skeletal tumours (red circled) were identified and confirmed by further anatomical examination post mortem up to 8 weeks post injection. $\mathbf{b}$ The number of skeletal tumours per mouse, $\mathbf{c}$ the number of non-skeletal tumour per mouse (skeletal muscle, liver, adrenal glands, but not peri- or intra-cardial tumours), and $\mathbf{d}$ the total tumour burden measured by radiance of luminescence were also compared between young and mature mice, $n=12$. e A 3D bone models created using the micro-CT scan on tumour bearing femurs in young mice shows the bone lesions caused by these tumours. $\mathbf{f}$ To measure the bone destruction in trabecular compartment, trabecular bone content (BV/TV) were then quantified and compared between tumour bearing and non-tumour bearing legs of young mice, $n>8$. (** $P<0.01,{ }^{* * * *} P<0.0001$, t-test)

located in close proximity (within a $50 \mu \mathrm{m}$ range) to bone at all time-points in both age groups, but were found to be located significantly closer to bone in young animals than in mature animals 1 day post-injection. This trend continued on day 7 but was not present at 3 weeks (Fig. 2c).

\section{Bone turnover was higher in young compared to} mature mice

As the number of tumour cells seeded into bone is unlikely to account for the increase in overt metastases in young animals, we explored the effects of changes in the bone microenvironment to this process. Using naïve 

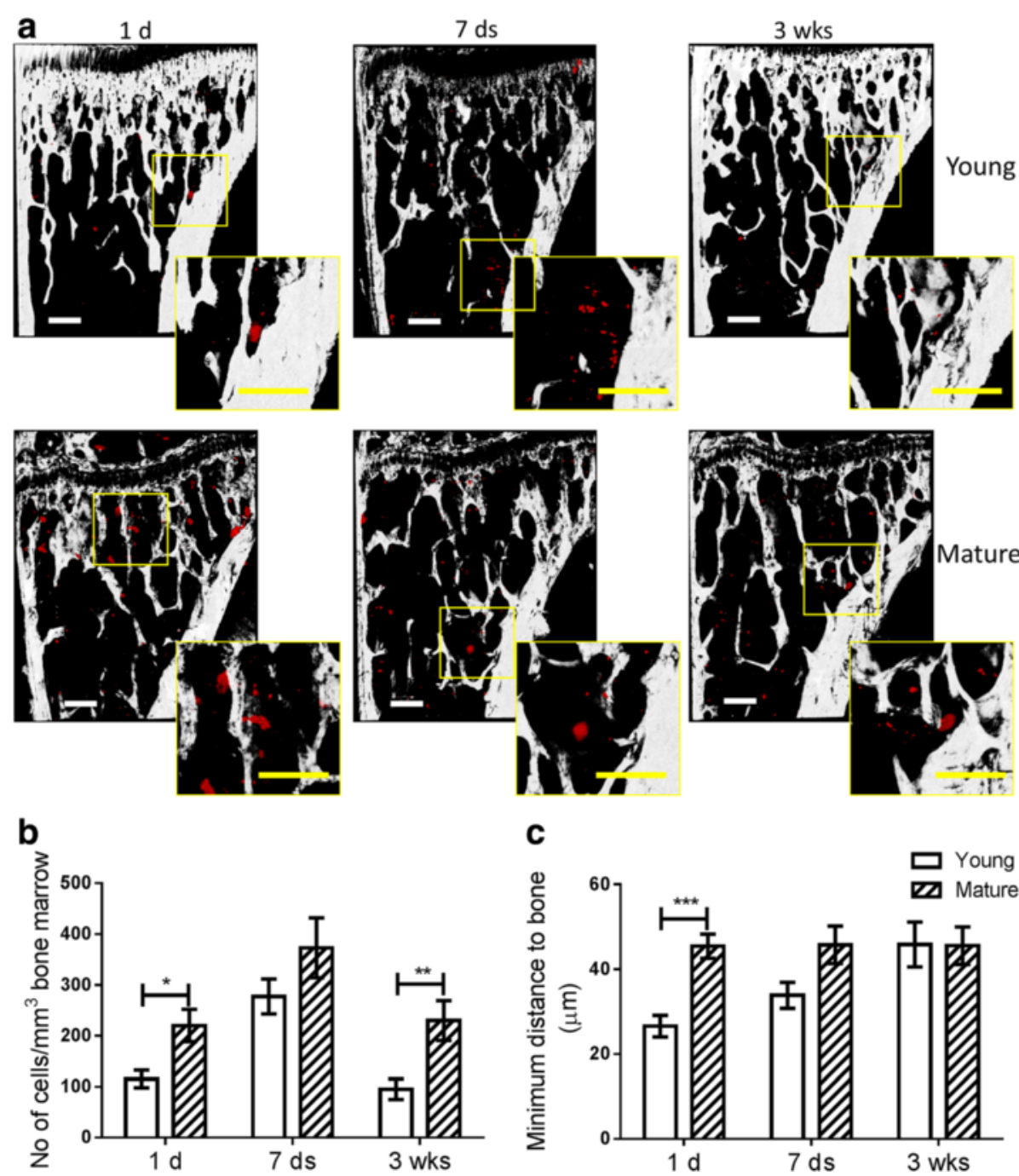

Fig. 2 Quantification of human prostate cancer cells arriving and colonizing the bone marrow of male mice. a The DiD labelled tumour cells entering the bone microenvironment were visualized and quantified using two-photon microscopy on day 1, 7 and 21 post tumour cells injection. Scale bar $=200 \mu \mathrm{m}$. b The number of tumour cells per $\mathrm{mm}^{3}$ bone marrow volume and $\mathbf{c}$ the minimum distance from tumour cells to the nearest bone surface was quantified and compared between 6-week old (Young) or 16-week old (Mature) mice using the Volocity 3D Image Analysis software. $n>6,{ }^{*} p<0.05,{ }^{*} p<0.01,{ }^{* * *} p<0.001$, t-test

mice, we carried out detailed micro-CT analysis on distal regions of femurs and showed that the young male mice had $18 \%$ lower $\mathrm{BV} / \mathrm{TV}$ compared to mature male mice $(17.4 \pm 0.9$ vs $21.2 \pm 1.2, p=0.0194)$ (Fig. $3 \mathrm{a}$ and $\mathrm{b})$. However, the younger mice have greater overall bone remodelling activities than older mice, with evidence of nearly 3 fold more bone formation $(134.3 \pm 17.0$ vs $34.5 \pm 1.7$ $\mathrm{ng} / \mathrm{mL}, p=0.0004)$ and increased bone resorption activities $(5.9 \pm 0.5$ vs $2.7 \pm 0.1 \mathrm{U} / \mathrm{mL}, p=0.0002)$, determined via quantifying serum P1NP and TRAP concentrations, respectively (Fig. 3c and d). This is consistent with histomorphometric analysis of the osteoblasts and TRAP positive osteoclasts on the endocortical bone surface of the tibiae (Fig. 3e). These data showed that young male mice had increased numbers of osteoblasts $(58.5 \pm 0.5 \mathrm{vs}$ $28.9 \pm 4.4 \mathrm{~mm}^{-1}, p=0.0024$ )(Fig. 3f), increased areas of osteoblast covered bone surfaces $(66.7 \pm 1.7$ vs $38.2 \pm 5.8 \%$, $p=0.0056$ ) (Fig. $3 \mathrm{~g}$ ), increased numbers of osteoclasts $\left(1.8 \pm 0.5\right.$ vs $\left.0.8 \pm 0.1 \mathrm{~mm}^{-1}, p=0.0127\right)$ (Fig. 3h), and 2 fold more osteoclast covered bone surfaces ( $4.5 \pm 1.2$ vs $1.5 \pm 0.2 \%, p=0.0187$ )(Fig. 3i) than mature animals.

In order to ensure that the above results were not unique to the prostate cancer model we repeated the studies using breast cancer cells injected in female mice, assessing tumour cell seeding in bone, progression of metastases and bone turnover. 


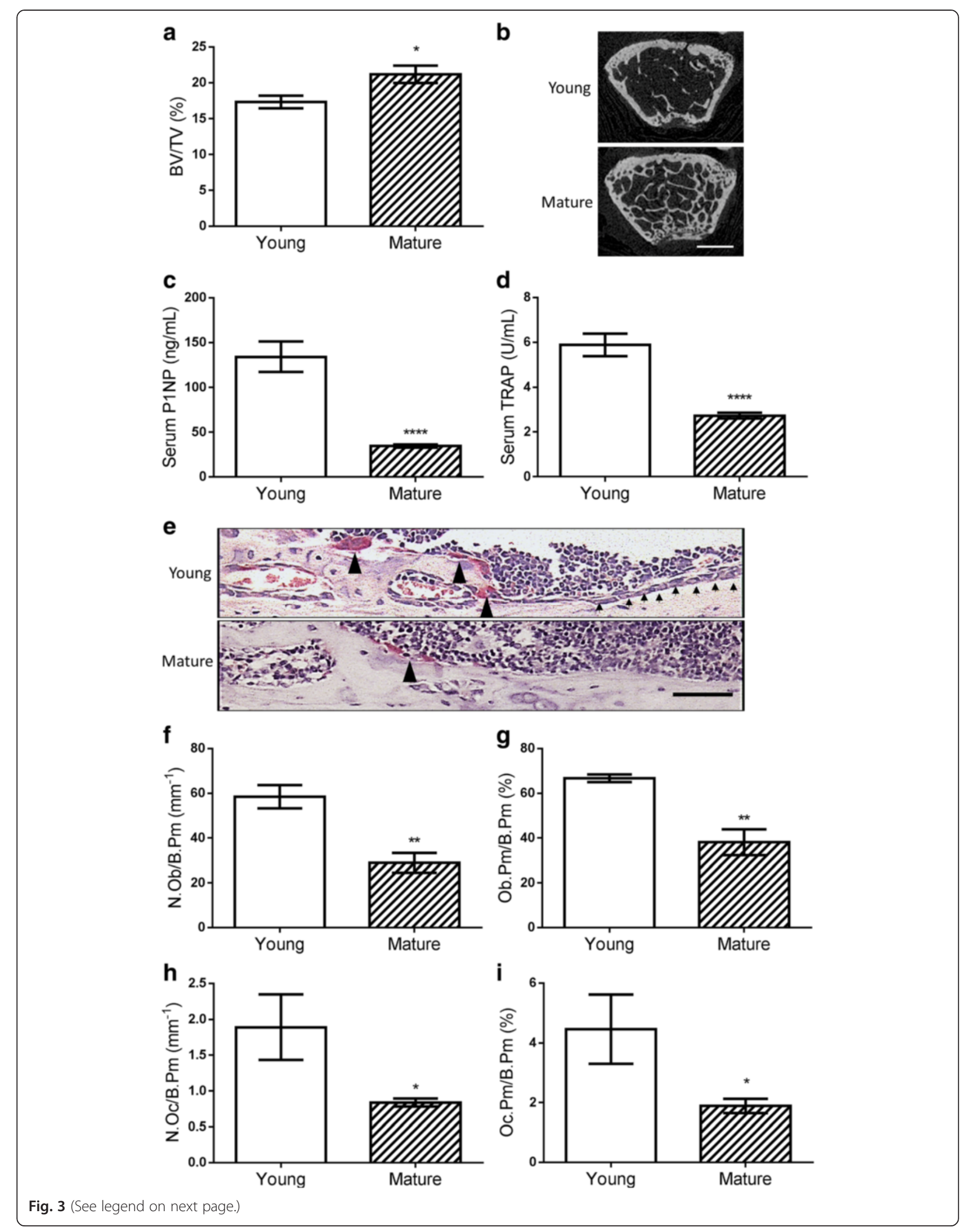


(See figure on previous page.)

Fig. 3 Differences of bone remodelling activities between younger and older male BALB/C nude mice. a The trabecular bone content (BV/TV) was compared between 6-week old (Young) or 16-week old (Mature) male mice using micro-CT analysis. $n>9$. b The trabecular bone mass difference can be clearly seen on the cross-section micro-CT image $250 \mu \mathrm{m}$ below the growth plate, scale bar $=500 \mu \mathrm{m}$. c The bone formation marker (P1NP) and d bone resorption marker (TRAP) were also measured in mouse serum sample using ELISA kits, $n>9$. e The bone resorbing osteoclasts (TRAP staining + , marked by black arrow heads) and bone forming osteoblasts (marked by small black arrows) were viewed and quantified on the endocortical bone surface of 6- and 16-week old mice tibiae after sectioning and TRAP staining. Scale bar $=50 \mu m$. $\mathbf{f}$ The number of osteoblasts per mm endocortical bone surface (N. Ob/B.Pm), g bone surface covered by osteoblasts (Ob.Pm/B.Pm), h number of osteoclasts per mm endocortical bone surface (N.Oc/B.Pm), and i bone surface covered by osteoclasts (Oc.Pm/B.Pm) were all compared between 6- and 16-week old mice. $n>4$, ${ }^{*} P<0.05,{ }^{* *} p<0.01,{ }^{* * * *} p<0.0001$, t-test

The frequency of overt, skeletal breast cancer metastases was elevated in young compared to mature female mice Three weeks after injection of DiD-labelled MDA-MB231 human breast cancer cells, young female mice had developed significantly higher numbers of tumours in the skeleton $(\sim 80 \%)$ compared to mature animals ( $\sim 50 \%$ skeletal tumours at both 3 and 8 weeks post injection) (Fig. 4a). The tumours caused lytic bone lesions in both young and mature mice, as shown in the 3D micro-CT images of tumour bearing femurs of young mice (Fig. 4b) and led to significant decrease in BV/TV (12.9 \pm 1.2 vs $6.7 \pm 1.7, p=0.0107)$ (Fig. 4c). Younger female mice carried an average of $4.8 \pm 0.5$ skeletal tumours compared to $0.7 \pm 0.2$ in older mice $(p<0.0001)$ (Fig. 4d). There was no difference in non- skeletal tumour frequency (skeletal muscle, liver, brain but not peri- or intra-cardial tumours) between the groups $(0.4 \pm 0.2$ vs $0.3 \pm 0.2, p=0.9087)$ (Fig. $4 \mathrm{e})$. The skeletal tumour burden in younger female mice was about 56 fold higher than that of older mice $(13470000 \pm 9121000$ vs $233787 \pm 82546 \mathrm{p} / \mathrm{sec} / \mathrm{cm} 2 / \mathrm{sr}$, $p=0.2373)$ (Fig. 4f).

\section{Similar numbers of breast cancer cells were present in bone of young and mature female mice}

To investigate whether different numbers of tumour cells seed to and colonize bone microenvironment of young and mature female mice, DiD labelled breast cancer cells were identified and quantified in tibial bone marrow at different time points ( 1 day, 7 days, and 3

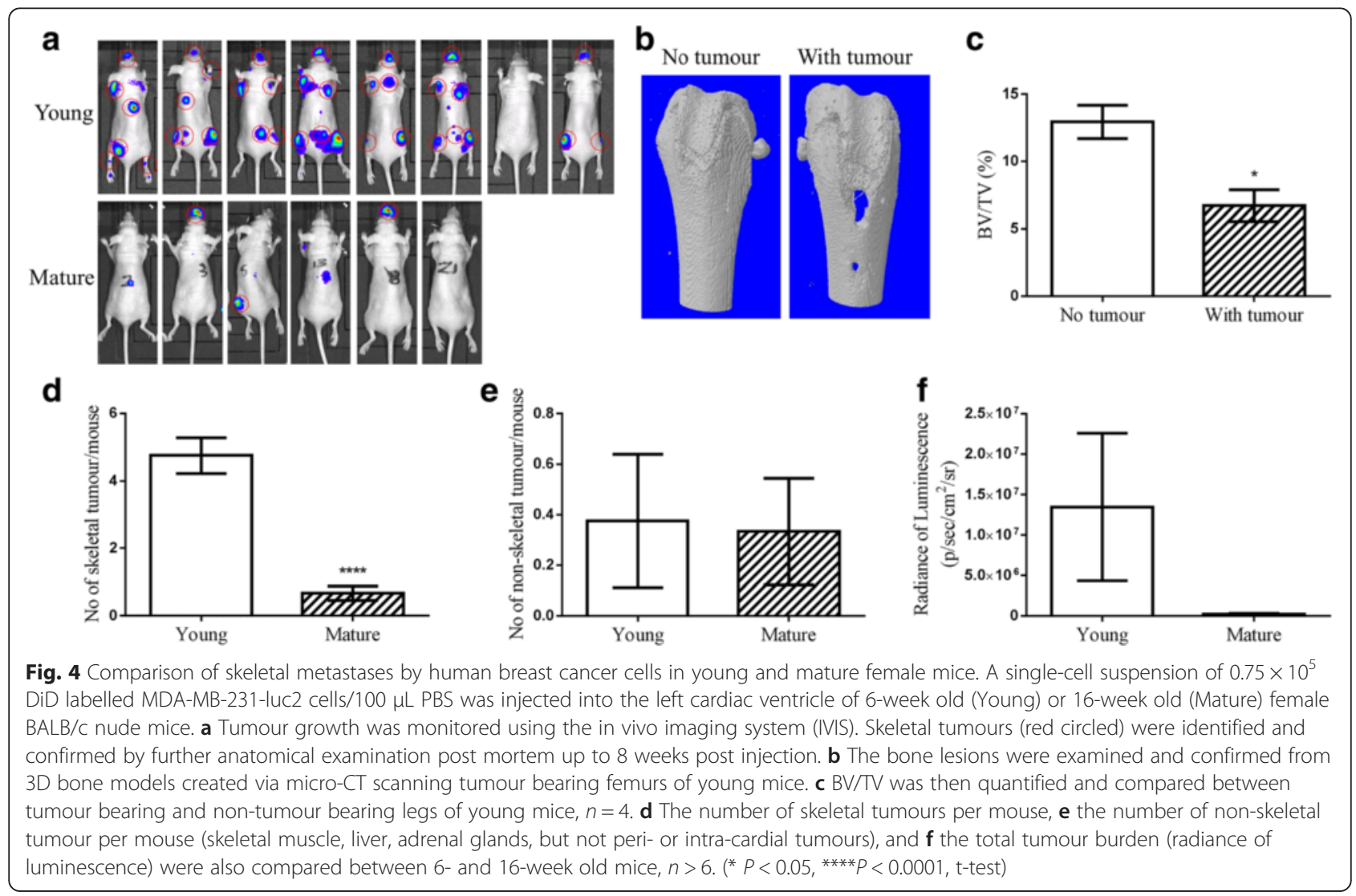



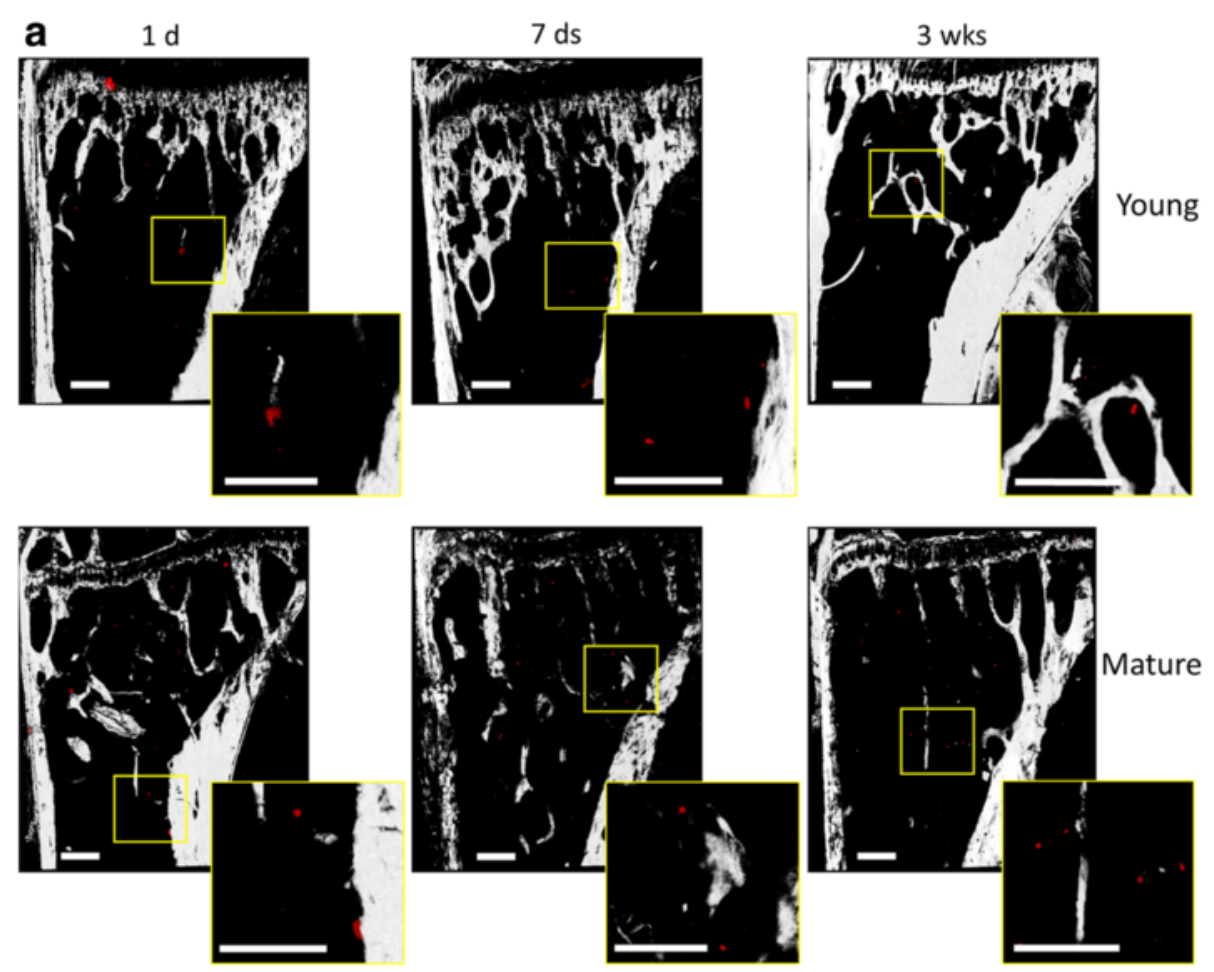

b

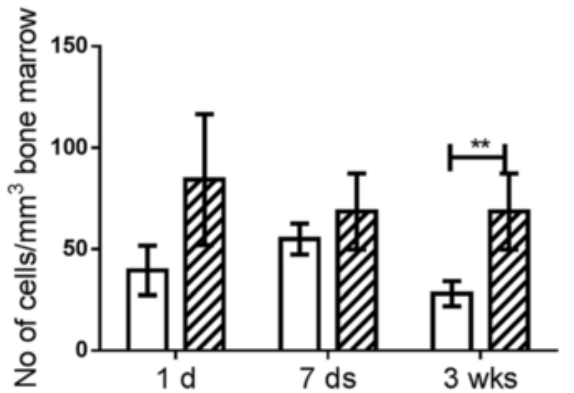

c

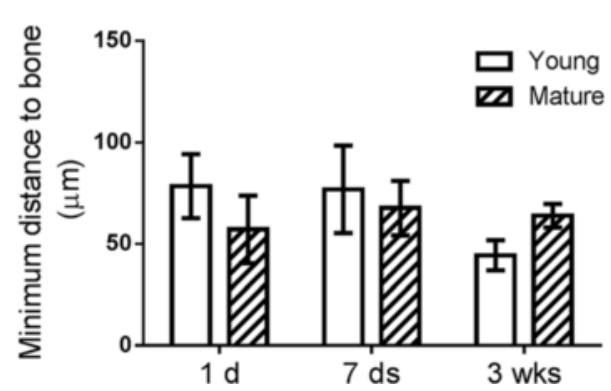

Fig. 5 Quantification of human breast cancer cells arriving and colonizing the bone marrow of female mice. a The DiD labelled tumour cells entering the bone microenvironment were visualized and quantified using a two-photon microscopy on day 1, 7 and 21 post tumour injection. Scale bar $=200 \mu \mathrm{m}$. $\mathbf{b}$ The number of tumour cells per $\mathrm{mm}^{3}$ bone marrow volume and $\mathbf{c}$ the minimum distance from tumour cells to bone surfaces were quantified and compared between 6 - and 16-week old mice using the Volocity $3 \mathrm{D}$ Image Analysis software. $n>6$, ${ }^{* *} P<0.01$, t-test

weeks post injection) using two-photon microscopy ex vivo (Fig. 5a). There were no significant differences between young and mature mice at 1 and 7 days post inject, while significantly fewer tumour cells were found in young animals 3 weeks post injection (Fig. 5b). There was also no difference between younger and older female mice in the distances from tumour cells to the nearest bone surfaces (Fig. 5c).

Bone turnover was higher in young compared to mature female mice

To evaluate differences in bone remodelling activities, detailed analyses of bone structure were performed and phenotypes were compared between the young and mature female mice. Micro-CT analysis on femurs showed that the young female mice had $18 \%$ less $\mathrm{BT} / \mathrm{TV}$ compared to mature animals $(7.7 \pm 0.5$ vs $12.2 \pm 0.6, p=0.0007$ )(Fig. $6 \mathrm{a}$ and $\mathrm{b}$ ). The young mice had evidence of higher bone formation (98.0 $\pm 3.2 \mathrm{vs}$ $36.7 \pm 6.7 \mathrm{ng} / \mathrm{mL}$ osteocalcin in serum samples, $p<$ 0.0001 )(Fig. 6c) and 4 fold greater bone resorption activity $(22.2 \pm 0.3$ vs $4.4 \pm 0.3 \mathrm{U} / \mathrm{mL}$ TRAP activity, $p<0.0001$ ) than the mature mice (Fig. 6d). Quantified at cellular level and compared to mature mice, younger female mice have $136 \%$ more osteoblasts $\left(74.4 \pm 5.8\right.$ vs $\left.31.5 \pm 7.6 \mathrm{~mm}^{-1}, p=0.0007\right)$ (Fig. 6e) and $89 \%$ more osteoblast covered bone surfaces $(72.1 \pm 4.3$ vs $38.2 \pm 8.8 \%, p=0.0028)$ (Fig. $6 f)$. In 

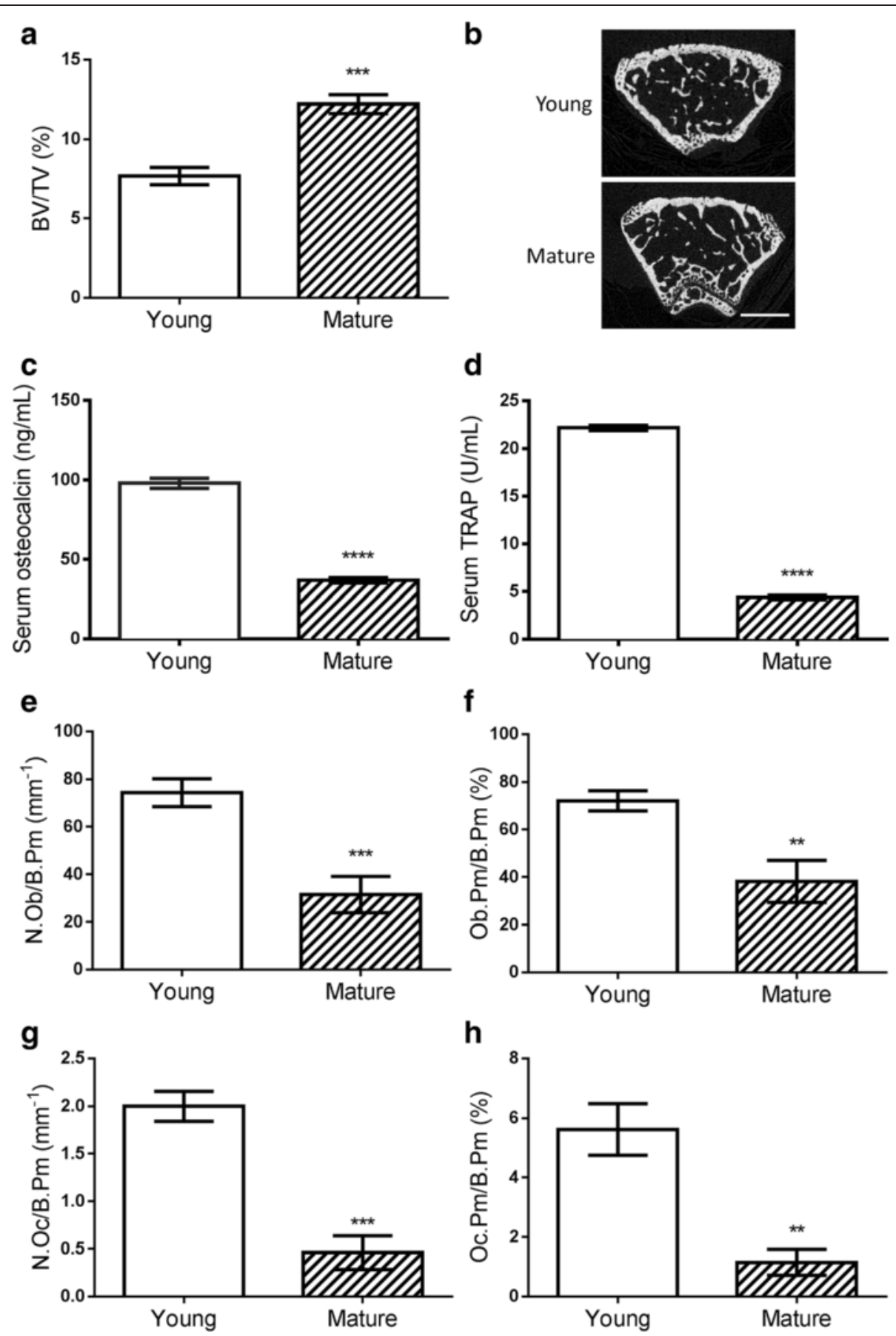

Fig. 6 Differences of bone remodelling activities between young and mature female BALB/c nude mice. $\mathbf{a} \& \mathbf{b}$ BV/TV was compared between 6-week old (Young) or 16-week old (Mature) female mice using the micro-CT analysis. $n>6$, scale bar $=500 \mu$ m. $\mathbf{c}$ The bone formation marker (Osteocalcin) and $\mathbf{d}$ bone resorption marker (TRAP) were also measured in mouse serum sample by ELISA, $n=5$. e The number of osteoblasts per $\mathrm{mm}$ endocortical bone surface (N. Ob/B.Pm), $\mathbf{f}$ bone surface covered by osteoblasts (Ob.Pm/B.Pm), $\mathbf{g}$ number of osteoclasts per mm endocortical bone surface (N.Oc/B.Pm), and $\mathbf{h}$ bone surface covered by osteoclasts (Oc.Pm/B.Pm) were all compared between young and mature mice. $n>4$, ${ }^{*} P<0.05,{ }^{*} P<0.01,{ }^{* * *} P<0.001,{ }^{* * * *} P<0.0001$, t-test

terms of osteoclasts, the young mice have $334 \%$ more osteoclasts $\left(2.0 \pm 0.2\right.$ vs $\left.0.5 \pm 0.2 \mathrm{~mm}^{-1}, p=0.0002\right)$ (Fig. $6 \mathrm{~g}$ ) and $389 \%$ more osteoclast covered bone surfaces $(5.6 \pm 0.9$ vs $1.2 \pm 0.4 \%, p=0.0018$ )(Fig. $6 \mathrm{~h}$ ).

\section{Discussion}

The 'Seeds and soils' hypothesis suggests that both the metastasis initiating tumour cells and the microenvironment are equally important for the initiation of 
metastases [8]. To test this, we used different aged (6week and 16-week old) immunocompromised mice as xenograft models to investigate the relationship between numbers of tumour cells colonizing bone, bone turnover, and the incidence of osteolytic skeletal metastases caused by prostate and breast cancer.

In the prostate cancer model, bioluminescent signals were clearly visible in the skeleton, three weeks after initial tumour cell injection. As bioluminescence detects bone marrow metastases of $\approx 0.5 \mathrm{~mm}^{3}$ volume [27], the signals detected were indicative of overt, growing lesions. These lesions were associated with bone destruction that was confirmed by anatomical confirmation and micro-CT analysis post mortem. The results showed that both the frequency of skeletal metastases and tumour burden were higher in young mice compare to mature mice. However, there were no differences in the incidence of metastases in soft tissues. This indicates that the difference in the frequency of metastases across different aged cohorts is unique to the skeletal system and determined by the bone microenvironment. Further examination of the bone phenotype showed that young male mice had lower bone mass compared to mature animals but higher bone turnover. This was characterised by significant increases in the numbers of osteoblasts and osteoclasts, and elevated bone formation and resorption activities. This is consistent with clinical studies which suggested higher levels of bone turnover predict poor outcome in patients with bone metastasis [20, 22,28 . To determine whether the higher metastasis frequency is due to higher numbers of metastasis initiating cells taking up residency in bone, we quantified the numbers of prostate cancer cells present in the bone marrow across a three-week period after initial injection, using two-photon microscopy and cell membrane labelling techniques. The results suggested that there were no increase in prostate cancer cells arriving in the bone marrow of young mice compared to mature animals and that these cells were in direct contact or one cell layer away from bone surface osteoblast lineage cells as they were within a range of $50 \mu \mathrm{m}$ to the nearest bone surface on which an osteoblast has a typical diameter of 15-30 $\mu \mathrm{m}$ [29]. Surprisingly, there were significantly more tumour cells detected in the bone marrow of mature mice on day 1 and at 3 weeks post injection. This warrants further investigation. One explanation is that fewer $\mathrm{DiD}$ labelled tumour cells were detected in younger mice as a result of more proliferation and consequent loss of dye [24]. Another possibility is that the low bone turnover microenvironment in mature mice is more selective for stem cell-like cancer cells, while high bone turnover microenvironment in younger mice are more supportive to the growth of metastases and may correlated with angiogenesis which will be discussed in following sections. These data suggest that the higher frequency of skeletal metastases for prostate cancer in younger xenograft models is not due to increased tumour cells homing to the bone marrow but supports the hypothesis that it is the bone microenvironment that controls the frequency of growing metastases detected in these xenograft models, through induction of lesion initiation and growth [30, 31].

Similar results were found in the breast cancer model. Significantly higher frequencies and levels of tumour burden, determined by bioluminescence and anatomical confirmation post-mortem, were found in young female mice compared to mature mice. This was not due to higher number of breast cancer cells arriving into the bone marrow of younger animals, but correlated with the enhanced bone turnover activities confirmed by both ELISA of serum bone turnover marker (osteocalcin and TRAP) and histomorphometric analysis of osteoblasts and osteoclasts. Once again, there was a trend towards more tumour cells being detected in bone marrow of older female mice.

Taken together, the differences in frequency of skeletal metastases between young and mature mice in both prostate and breast cancer models strongly suggested that it is the trigger for the proliferation of resident tumour cells in bone that is linked to the differences in bone turnover in the bone microenvironment. This is consistent with previous findings of our own and of others that showed increased frequencies of bone metastases by prostate and breast cancer xenograft models in mature animals with experimentally induced enhancement of bone turnover [17, 18, 32]. More importantly, these data correlate with disease outcomes in cancer patients: Breast cancer patients presenting at a younger age $(<40)$ have worse survival rates and more metastases compared to disease presented in older women, while younger men $(<44)$ with high grade prostate cancer, have poorer prognosis compared to older men with a similar grade/stage distribution $[33,34]$. One could argue these differences are due to higher available sex steroid hormone levels rather than the higher bone turnover in younger populations, directly affecting tumour growth. However, our data suggests another mechanism, as we intentionally used sex steroid hormone-independent cell lines: PC3 and MDA-MB-231 in these studies that are unaffected by androgens/oestrogens.

The xenograft model used in this study has limitations: firstly, we could not determine the role of the immune system, specifically $\mathrm{T}$ cells, in regulating metastatic frequency. The latter have been implicated in the bone metastatic process [35]. Secondly, the administration of a single high dose of cancer cells via intracardiac injection only partially mirrors seeding events in humans where low numbers of cancer cells are released into the circulation over prolonged time intervals. Finally, other 
differences between young and mature mice could also contribute to variations in metastatic frequencies such as the higher growth hormone levels and continuous longitudinal bone growth in younger mice. Particularly, differences in endothelial lineage populations and associated niches could also affect metastasis frequency and should be investigated [16]. Tumour cells were shown to be rapidly engrafted into bone marrow endothelial microdomains, where endothelial cells express higher level of adhesion molecules such as E-selectin, Pselectin, and intercellular adhesion molecule (ICAM-1) [36-38]. Kusumbe et al. recently showed that there are two distinct bone microvessels based on the expression of CD31 (also known as PECAM1) and Endomucin (Emcn), which were proposed as type $\mathrm{H}$ subset $\left(\mathrm{CD} 31^{\mathrm{hi}} \mathrm{Emcn}^{\mathrm{hi}}\right)$ and type L subset $\left(\mathrm{CD} 31^{\mathrm{lo}} \mathrm{Emcn}^{\mathrm{lo}}\right)$ [39]. The type $\mathrm{H}$ subset expresses higher level of metastasis related growth factor transcripts such as $T g f b 1$ and $\mathrm{Tg} f b 3$ [40]. The type $\mathrm{H}$ subset is a key to couple angiogenesis to osteogenesis and was strongly reduced in bone from aged animals [39]. This study suggested that the correlation of higher incidence of skeletal tumours in younger animals with the presence of type $\mathrm{H}$ microvessels in addition to higher bone turnover.

In conclusion, our study using two established metastatic cancer cell lines, provides further evidence to support the hypothesis that the frequency of overt skeletal metastases in hormone-independent prostate and breast cancer is determined by growth initiating influences within the bone microenvironment not by numbers of tumour cell initially seeding to these sites and provides further grounds for the use of treatments to suppress bone turnover and prevent development of skeletal metastases of prostate and breast cancer at early stages of disease.

\section{Abbreviations}

BV/TV: Trabecular bone content; IVIS: In vivo imaging system; N.Ob/ B.Pm: Number of osteoblasts; N.Oc/B.Pm: Number of osteoclasts; Ob.Pm/ B.Pm: Bone surface covered by osteoblasts; Oc.Pm/B.Pm: Bone surface covered by osteoclasts; P1NP: Type 1 procollagen amino-terminal-propeptide; SRE: Skeletal-related events; TRAP: Tartrate-resistant acid phosphatase 5b.

\section{Competing interests}

The authors declare that they have no competing interests.

\section{Authors' contributions \\ Study design: NW, KR, PC, IH, and CE. Study conduct: NW, AF, KR. Data acquisition: NW, FD, AF. Data analysis: NW, HB, and CE. Data interpretation: NW, PO, PC, IH and CE. Drafting manuscript: NW and CE. Revising manuscript content: NW, HB, PO, PC, $\mathrm{H}$, and CE. Approving final version of manuscript: $P C, I H$, and CE. NW and CE take responsibility for the integrity of the data analysis. All authors read and approved the final manuscript.}

\section{Acknowledgement}

The authors wish to thank Cancer Research UK for their generous financial support and Yorkshire Cancer Research for funding in vivo imaging equipment (IVIS). PIC is supported by Mrs Janice Gibson and the Ernest Heine Family Foundation and the Prostate Cancer Foundation of Australia and Movember, through a Movember Revolutionary Team Award. We are grateful for the expert technical support from Miss Orla Gallagher, Mr Mark Kinch and Mr Darren Lath.

\section{Author details}

${ }^{1}$ The Mellanby Centre for Bone Research, Department of Human Metabolism, Medical School, University of Sheffield, Beech Hill Road, Sheffield S10 2RX UK. ${ }^{2}$ Department of Oncology, Medical School, University of Sheffield, Sheffield, UK. ${ }^{3}$ Break Through Breast Cancer Research Unit, Paterson Institute for Cancer Research Manchester, Manchester, UK. ${ }^{4}$ Bone Biology Division, Garvan Institute of Medical Research, Sydney, Australia.

Received: 7 July 2015 Accepted: 12 October 2015

Published online: 20 October 2015

\section{References}

1. Chen YC, Sosnoski DM, Mastro AM. Breast cancer metastasis to the bone: mechanisms of bone loss. Breast Cancer Res. 2010;12(6):215.

2. Bubendorf L, Schopfer A, Wagner U, Sauter G, Moch H, Willi N, et al. Metastatic patterns of prostate cancer: an autopsy study of 1,589 patients. Hum Pathol. 2000;31(5):578-83.

3. Santoni M, Conti A, Procopio G, Porta C, Ibrahim T, Barni S, et al. Bone metastases in patients with metastatic renal cell carcinoma: are they always associated with poor prognosis? J Exp Clin Cancer Res. 2015;34:10.

4. Facchini G, Caraglia M, Santini D, Nasti G, Ottaiano A, Striano S, et al. The clinical response on bone metastasis from breast and lung cancer during treatment with zoledronic acid is inversely correlated to skeletal related events (SRE). J Exp Clin Cancer Res. 2007;26(3):307-12.

5. Coleman RE, Marshall H, Cameron D, Dodwell D, Burkinshaw R, Keane M, et al. Breast-cancer adjuvant therapy with zoledronic acid. N Engl J Med. 2011;365(15):1396-405.

6. Gnant M, Mlineritsch B, Stoeger H, Luschin-Ebengreuth G, Heck D, Menzel C, et al. Adjuvant endocrine therapy plus zoledronic acid in premenopausal women with early-stage breast cancer: 62-month follow-up from the ABCSG-12 randomised trial. Lancet Oncol. 2011;12(7):631-41.

7. Polascik TJ, Mouraviev V. Zoledronic acid in the management of metastatic bone disease. Ther Clin Risk Manag. 2008;4(1):261-8.

8. Paget $\mathrm{S}$. The distribution of secondary growths in cancer of the breast. 1889. Cancer Metastasis Rev. 1989;8(2):98-101.

9. Collins AT, Berry PA, Hyde C, Stower MJ, Maitland NJ. Prospective identification of tumorigenic prostate cancer stem cells. Cancer Res. 2005;65(23):10946-51.

10. Colombel M, Eaton CL, Hamdy F, Ricci E, van der Pluijm G, Cecchini M, et al. Increased expression of putative cancer stem cell markers in primary prostate cancer is associated with progression of bone metastases. Prostate. 2012;72(7):713-20.

11. Eaton CL, Colombel M, van der Pluijm G, Cecchini M, Wetterwald A, Lippitt $J$, et al. Evaluation of the frequency of putative prostate cancer stem cells in primary and metastatic prostate cancer. Prostate. 2010;70(8):875-82.

12. Liu H, Patel MR, Prescher JA, Patsialou A, Qian D, Lin J, et al. Cancer stem cells from human breast tumors are involved in spontaneous metastases in orthotopic mouse models. Proc Natl Acad Sci U S A. 2010;107(42):18115-20.

13. Guler G, Balci S, Costinean S, Ussakli CH, Irkkan C, Suren D, et al. Stem cell-related markers in primary breast cancers and associated metastatic lesions. Mod Pathol. 2012;25(7):949-55.

14. van der Horst $\mathrm{G}$, van den Hoogen C, Buijs JT, Cheung H, Bloys H, Pelger RC, et al. Targeting of alpha(v)-integrins in stem/progenitor cells and supportive microenvironment impairs bone metastasis in human prostate cancer. Neoplasia. 2011;13(6):516-25.

15. Wang N, Docherty FE, Brown HK, Reeves K, Fowles AC, Ottewell PD, et al. Prostate cancer cells preferentially home to osteoblast-rich areas in the early stages of bone metastasis - evidence from in vivo models. J Bone Miner Res. 2014;29(2):2688-96.

16. Shiozawa Y, Pedersen EA, Havens AM, Jung Y, Mishra A, Joseph J, et al. Human prostate cancer metastases target the hematopoietic stem cell niche to establish footholds in mouse bone marrow. J Clin Invest. 2011;121(4):1298-312.

17. Ottewell PD, Wang N, Meek J, Fowles CA, Croucher PI, Eaton CL, et al. Castration-induced bone loss triggers growth of disseminated prostate cancer cells in bone. Endocr Relat Cancer. 2014;21(5):769-81.

18. Ottewell PD, Wang N, Brown HK, Reeves KJ, Fowles CA, Croucher PI, et al. Zoledronic acid has differential antitumor activity in the pre- and 
postmenopausal bone microenvironment in vivo. Clin Cancer Res. 2014;20(11):2922-32.

19. Smith MR, Saad F, Coleman R, Shore N, Fizazi K, Tombal B, et al. Denosumab and bone-metastasis-free survival in men with castration-resistant prostate cancer: results of a phase 3, randomised, placebo-controlled trial. Lancet. 2012;379(9810):39-46.

20. Lipton A, Chapman JA, Demers L, Shepherd LE, Han L, Wilson CF, et al. Elevated bone turnover predicts for bone metastasis in postmenopausal breast cancer: results of NCIC CTG MA.14. J Clin Oncol. 2011;29(27):3605-10.

21. Stopeck AT, Lipton A, Body JJ, Steger GG, Tonkin K, de Boer RH, et al. Denosumab compared with zoledronic acid for the treatment of bone metastases in patients with advanced breast cancer: a randomized, double-blind study. J Clin Oncol. 2010;28(35):5132-9.

22. Fizazi K, Carducci M, Smith M, Damiao R, Brown J, Karsh L, et al. Denosumab versus zoledronic acid for treatment of bone metastases in men with castration-resistant prostate cancer: a randomised, double-blind study. Lancet. 2011;377(9768):813-22.

23. Yumoto K, Berry JE, Taichman RS, Shiozawa Y. A novel method for monitoring tumor proliferation in vivo using fluorescent dye DiD. Cytometry A. 2014;85(6):548-55.

24. Wang N, Docherty F, Brown HK, Reeves K, Fowles A, Lawson M, et al. Mitotic quiescence, but not unique "stemness," marks the phenotype of bone metastasis-initiating cells in prostate cancer. FASEB J. 2015;29(8):3141-50.

25. Bouxsein ML, Boyd SK, Christiansen BA, Guldberg RE, Jepsen KJ, Muller R. Guidelines for assessment of bone microstructure in rodents using micro-computed tomography. J Bone Miner Res. 2010;25(7):1468-86.

26. Wang N, Robaye B, Agrawal A, Skerry TM, Boeynaems JM, Gartland A. Reduced bone turnover in mice lacking the P2Y(13) receptor of ADP. Mol Endocrinol. 2012;26(1):142-52.

27. Wetterwald A, van der Pluijm G, Que I, Sijmons B, Buijs J, Karperien M, et al. Optical imaging of cancer metastasis to bone marrow: a mouse model of minimal residual disease. Am J Pathol. 2002;160(3):1143-53.

28. Roodman GD. High bone turnover markers predict poor outcome in patients with bone metastasis. J Clin Oncol. 2005;23(22):4821-2.

29. Jayakumar P, Di Silvio L. Osteoblasts in bone tissue engineering. Proc Inst Mech Eng H. 2010;224(12):1415-40.

30. Zheng Y, Zhou H, Brennan K, Blair JM, Modzelewski JR, Seibel MJ, et al. Inhibition of bone resorption, rather than direct cytotoxicity, mediates the anti-tumour actions of ibandronate and osteoprotegerin in a murine model of breast cancer bone metastasis. Bone. 2007;40(2):471-8.

31. Zhang J, Dai J, Qi Y, Lin DL, Smith P, Strayhorn C, et al. Osteoprotegerin inhibits prostate cancer-induced osteoclastogenesis and prevents prostate tumor growth in the bone. J Clin Invest. 2001;107(10):1235-44.

32. Schneider A, Kalikin LM, Mattos AC, Keller ET, Allen MJ, Pienta KJ, et al. Bone turnover mediates preferential localization of prostate cancer in the skeleton. Endocrinology. 2005;146(4):1727-36.

33. Anders CK, Johnson R, Litton J, Phillips M, Bleyer A. Breast cancer before age 40 years. Semin Oncol. 2009;36(3):237-49.

34. Lin DW, Porter M, Montgomery B. Treatment and survival outcomes in young men diagnosed with prostate cancer: a Population-based Cohort Study. Cancer. 2009;115(13):2863-71.

35. Capietto AH, Faccio R. Immune regulation of bone metastasis. Bonekey Rep. 2014;3:600.

36. Bussard KM, Gay CV, Mastro AM. The bone microenvironment in metastasis; what is special about bone? Cancer Metastasis Rev. 2008;27(1):41-55.

37. Sipkins DA, Wei X, Wu JW, Runnels JM, Cote D, Means TK, et al. In vivo imaging of specialized bone marrow endothelial microdomains for tumour engraftment. Nature. 2005;435(7044):969-73.

38. Bianco P. Bone and the hematopoietic niche: a tale of two stem cells. Blood. 2011;117(20):5281-8

39. Kusumbe AP, Ramasamy SK, Adams RH. Coupling of angiogenesis and osteogenesis by a specific vessel subtype in bone. Nature. 2014;507(7492):323-8.

40. Padua D, Massague J. Roles of TGFbeta in metastasis. Cell Res. 2009;19(1):89-102.

\section{Submit your next manuscript to BioMed Central and take full advantage of:}

- Convenient online submission

- Thorough peer review

- No space constraints or color figure charges

- Immediate publication on acceptance

- Inclusion in PubMed, CAS, Scopus and Google Scholar

- Research which is freely available for redistribution

Submit your manuscript at www.biomedcentral.com/submit 